\title{
Benefits may not outweigh risks of low molecular weight heparin (LMWH) in early postoperative thromboprophylaxis following minimally invasive cardiac surgery: a propensity score-matched analysis
}

\author{
Wei Li ${ }^{1,2 \#}$, Pei Wang ${ }^{1 \#}$, Shiguan Le ${ }^{1,3}$, Wang Xi ${ }^{1}$, Jing Wang ${ }^{1}$, Liang Yin ${ }^{1}$, Qing Wang ${ }^{1}$, Yufeng Zhang ${ }^{1}$, \\ Zhinong Wang ${ }^{1}$
}

${ }^{1}$ Department of Cardiothoracic Surgery, Changzheng Hospital, Second Military Medical University, Shanghai 200003, China; ${ }^{2}$ Department of Cardiothoracic Surgery, General Hospital of Central Theater Command, Wuhan 430012, China; ${ }^{3}$ Department of Surgery, 69220 Hospital of Chinese People's Liberation Army, Kuqa County 842000, China

Contributions: (I) Conception and design: W Li; (II) Administrative support: Z Wang, Y Zhang; (III) Surgical procedure performance: Z Wang, Y Zhang, W Li, P Wang, S Le, J Wang, L Yin; (IV) Collection and assembly of data: W Li, W Xi; (V) Data analysis and interpretation: W Li, P Wang, Q Wang; (VI) Manuscript writing: All authors; (VII) Final approval of manuscript: All authors.

\#These authors contributed equally to this study.

Correspondence to: Yufeng Zhang, MD; Zhinong Wang, MD. 31st Floor, 415\#, Rd. Fengyang, Changzheng Hospital, Shanghai 200003 , China.

Email: zhyf19810824@163.com; wangzn007@163.com.

Background: Whether the benefits of early prophylactic anticoagulation by low molecular weight heparin (LMWH) would outweigh its possible harms in patients after minimally invasive cardiac surgery (MICS) remains contentious. The aims of this study were to define the incidence of venous thromboembolism (VTE) and to assess whether early prophylactic anticoagulation by LMWH postoperatively was indeed effective in reducing VTE without increasing risk of complications after MICS.

Methods: This investigation was a single-center, retrospective, propensity score-matched analysis study. A total of 473 patients underwent MICS, of whom 257 received prophylactic anticoagulation with LMWH (LMWH group) in the early postoperative period and 216 were not treated with LMWH (Control group). The main outcome measurements included the incidence of embolism events and major bleeding events, the volume of erythrocyte transfusion, the volume of drainage and the duration of drainage after MICS. In addition, the incidence of poor wound healing, the mechanical ventilation time, ICU stay time and postoperative hospitalization time were also documented.

Results: There were fewer embolic events $(\mathrm{P}=1.000)$ and a higher rate of major bleeding events $(\mathrm{P}=0.008)$ in the LMWH group than the Control group, and their magnitude and significance were maintained in the propensity matched analysis. In the matched cohorts, there was no significant difference in the total volume of red blood cell transfusion $(\mathrm{P}=0.552)$, assisted mechanical ventilation time $(\mathrm{P}=0.542)$, and the ICU stay time $(\mathrm{P}=0.166)$ between the two groups; while the volume of drainage $(\mathrm{P}<0.001)$ and the duration of drainage $(\mathrm{P}<0.001)$ in the LMWH group were significantly more than the Control group, and the incidence of poor wound healing $(\mathrm{P}=0.009)$ and the postoperative hospitalization time $(\mathrm{P}<0.001)$ were significantly increased in the LMWH group.

Conclusions: Early prophylactic anticoagulation with LMWH could not reduce the incidence of embolism events after MICS. Instead, it might increase postoperative major bleeding events and prolong drainage tube indwelling time and the length of hospital stay.

Keywords: Minimally invasive surgery; cardiac surgical procedures; heparin; low-molecular-weight; venous thrombosis; blood transfusion; drainage 
Submitted Jun 20, 2019. Accepted for publication Nov 08, 2019.

doi: $10.21037 /$ jtd.2019.11.56

View this article at: http://dx.doi.org/10.21037/jtd.2019.11.56

\section{Introduction}

Heart valve disease remains a significant contributor to cardiac morbidity and mortality (1). Surgical correction with either repair or replacement is recommended for patients with low risk for surgery (2). In recent years, minimally invasive cardiac surgery (MICS) has evolved significantly in major cardiac centers worldwide, which has potential advantages such as less blood loss, lower complication rates, faster recovery and better cosmetic results (3-5). One of the most characteristic technical strategies in MICS is to establish extracorporeal circulation via peripheral arterial-venous cannulation. In the absence of significant aortoiliac disease, femoral arterial and venous cannulation is the preferred choice of many cardiac surgeons for MICS procedures (6). It offers convenience and maximal utilization of space provided by using smaller incisions (7). However, incision or percutaneous femoral vein cannulation may cause venous endothelial injury and abnormal blood stasis. According to Virchow's triad (8), endothelial injury and abnormal blood stasis are initiating factors of deep venous thrombosis (DVT). In addition, some other factors may increase the risk of DVT in patients underwent cardiac surgery including older age, postoperative complications, prolonged preoperative hospitalization or postoperative recovery (9), and so on. However, the occurrence of venous thromboembolism (VTE) after cardiac surgery cannot be prevented by intraoperative anticoagulation $(10,11)$, especially for MICS via peripheral arterial-venous cannulation.

The practice of early pharmacological VTE prophylaxis after cardiac surgery is controversial (12). Some recommended prophylactic anticoagulation should commence on the first postoperative day (13), while others suggested prescribing low molecular weight heparin (LMWH) or low-dose unfractionated heparin to prolonged hospitalization patients with non-bleeding complications, due to the evidence-based clinical practice guidelines on antithrombotic therapy and prevention of thrombosis, that classified most patients undergoing cardiac surgery as being at moderate risk for VTE and at high risk for major bleeding complications (14), which may increase the risk of massive bleeding and pericardial tamponade (15).
LMWH is a heparin fragment with a low molecular weight (average molecular weight of 4,000-6,000 D) degraded from unfractionated heparin, which is widely recommended for prevention and treatment of pulmonary embolism (PE) as well as DVT and recurrence $(16,17)$, due to its better bioavailability, more easily absorbed by subcutaneous injection, longer half-life, less adverse reactions, and better predictability of clinical effects $(18,19)$.

However, there are only few studies revealed whether the benefits of early postoperative prophylactic anticoagulation with LMWH outweighed the potential harms for patients undergoing MICS. This study aimed to define the incidence of VTE including DVT and PE, and to assess whether early prophylactic anticoagulation with LMWH postoperatively increased risk of complications after MICS.

\section{Methods}

\section{Patient selection and data collection}

The study was a retrospective cohort study. Data of patients underwent MICS between January 2012 and December 2016 at Department of Cardiothoracic Surgery, Changzheng Hospital, Second Military Medical University were collected. The study protocol was approved by Committee on Ethic of Biomedicine Research, Second Military Medical University (No. 2011SL037), and met the ethical and legal requirements. All subjects have signed informed consent for surgery, perioperative therapy and related medical research. MICS patients with complete medical records were enrolled. Patients with infective endocarditis, a history of severe coagulopathy, active bleeding after surgery and bleeding-prone organ damage were excluded. Detailed medical information of enrolled patients was obtained from our hospital database. Among 473 enrolled patients, 257 patients $(54.3 \%)$ received LMWH (LMWH group) in the early postoperative period and the rest were not treated with LMWH (Control group).

\section{Definition}

Preoperative comorbidities were defined according to the Euro SCORE risk stratification model (20). Postoperative 
DVT referred to a new blood clot or thrombus that occurs postoperatively in the venous system, and the diagnosis was based on highly suspicious clinical presentations and was identified by Doppler ultrasonography. The diagnosis of postoperative PE was based on highly suspicious clinical presentations and was identified by chest computed tomography. Major bleeding event was defined as any of the following: proved fatal bleeding, intracranial hemorrhage (based on highly suspicious clinical presentations and identified by cerebral computed tomography or magnetic resonance scan), bleeding requiring an intervention (hemopericardium requiring re-exploration or catheter drainage, hemothorax requiring thoracotomy or chest tube, gastrointestinal bleeding requiring surgery or endoscopic treatment, wound bleeding requiring reoperation). The volume of red blood cell transfusion and the volume of drainage were respectively defined as the total volume of red blood cell transfusion, pericardial drainage and pleural drainage from the 6 hours after surgery. Postoperative drainage tube indwelling time referred to the time between drainage tube insertion and extubation. Impaired wound healing referred to wound infection or subcutaneous fat liquefaction requiring non-pharmacological intervention.

\section{Anesthesia, operation and postoperative management}

Superior partial median sternotomy or right anterior minithoracotomy incision was performed in isolated aortic valve replacement; right anterolateral mini-thoracotomy or inferior partial median sternotomy incision was applied in isolated mitral valve repair/replacement, isolated tricuspid valve repair, or mitral valve repair/replacement concomitant with tricuspid valve repair. Choices of surgical approach were decided by the surgeons. Systemic heparinization was performed according to adjusted calculation model of heparin doses as described previously (21). Extracorporeal circulation was established via femoral arterial-venous cannulation. Myocardial protection was performed by aortic root antegrade perfusion and/or coronary sinus retrograde perfusion. One milligram of protamine was used to neutralize $100 \mathrm{U}$ of heparin at the end of extracorporeal circulation, and was added according to activated clotting time. All patients in the LMWH group received a subcutaneous injection of $40 \mathrm{mg}$ of enoxaparin (Clexane, SANOFI WINTHROP INDUSTRIE, France) once daily, the initial dose of which was generally administered 6 hours postoperatively. Warfarin anticoagulation was initiated after removal of the chest tube in patients undergoing valve replacement surgery. For patients receiving warfarin, enoxaparin was continued to be applied until meeting international normalized ratio target value. For patients who did not receive warfarin, enoxaparin was applied up to the start of ambulation. Postoperative transfusion triggers were hemoglobin $\leq 7 \mathrm{~g} / \mathrm{dL}$ and/or hematocrit $\leq 28 \%$. The chest tube could be removed as the 24-hour total drainage volume was less than $150 \mathrm{~mL}$.

\section{Statistical analysis}

All continuous variables were expressed as mean \pm standard deviation and count variables were expressed as frequency (percentage). Pearson's Chi-square or Fisher's exact Chisquare tests were used to compare count variables between the two groups. Unpaired Student's $t$ test was used for normally distributed continuous variables and MannWhitney $\mathrm{U}$ test was used for non-normally distributed continuous variables. All tests were two-tailed. A P value $<0.05$ was considered statistically significant.

Propensity-score matching (PSM) was used to compare matched study cohorts between the LMWH group and the control group in order to reduce the impact of selection bias and potential confounders (22). A multivariate logistic regression analysis was used to determine the propensity of MICS patients' postoperative LMWH application. Propensity score representing the likelihood of receiving LMWH treatment was calculated by the logistic equation. A 1:1 nearest neighbor matching (with a caliper width of 0.2 ) was used to generate study cohorts with equal sample sizes. Statistical analysis was performed on IBM SPSS Statistics, version 22.0 (SPSS, Inc., Armonk, NY, USA) and $\mathrm{R}$ statistical software, version 2.15.1 (The R Foundation: http://www.R-project.org).

\section{Results}

In the matched cohorts, no significant covariate difference was found between the two groups (Table 1).

Before PSM, there were fewer embolic events in the LMWH group, though the difference was not statistically significant $(0.4 \%$ vs. $1.4 \%, \mathrm{P}=1.000)$. There were only 1 embolic event (DVT) in the LMWH group and 3 embolic events (1 shock and 2 DVT) in the Control group. There was a significant higher rate of major bleeding events in the LMWH group than in the Control group $(16.0 \%$ vs. $7.9 \%, \mathrm{P}=0.008)$. There were 41 major bleeding events (14 hemopericardium and 27 hemothorax) in the LMWH 
Table 1 Patient characteristics before and after propensity score matching

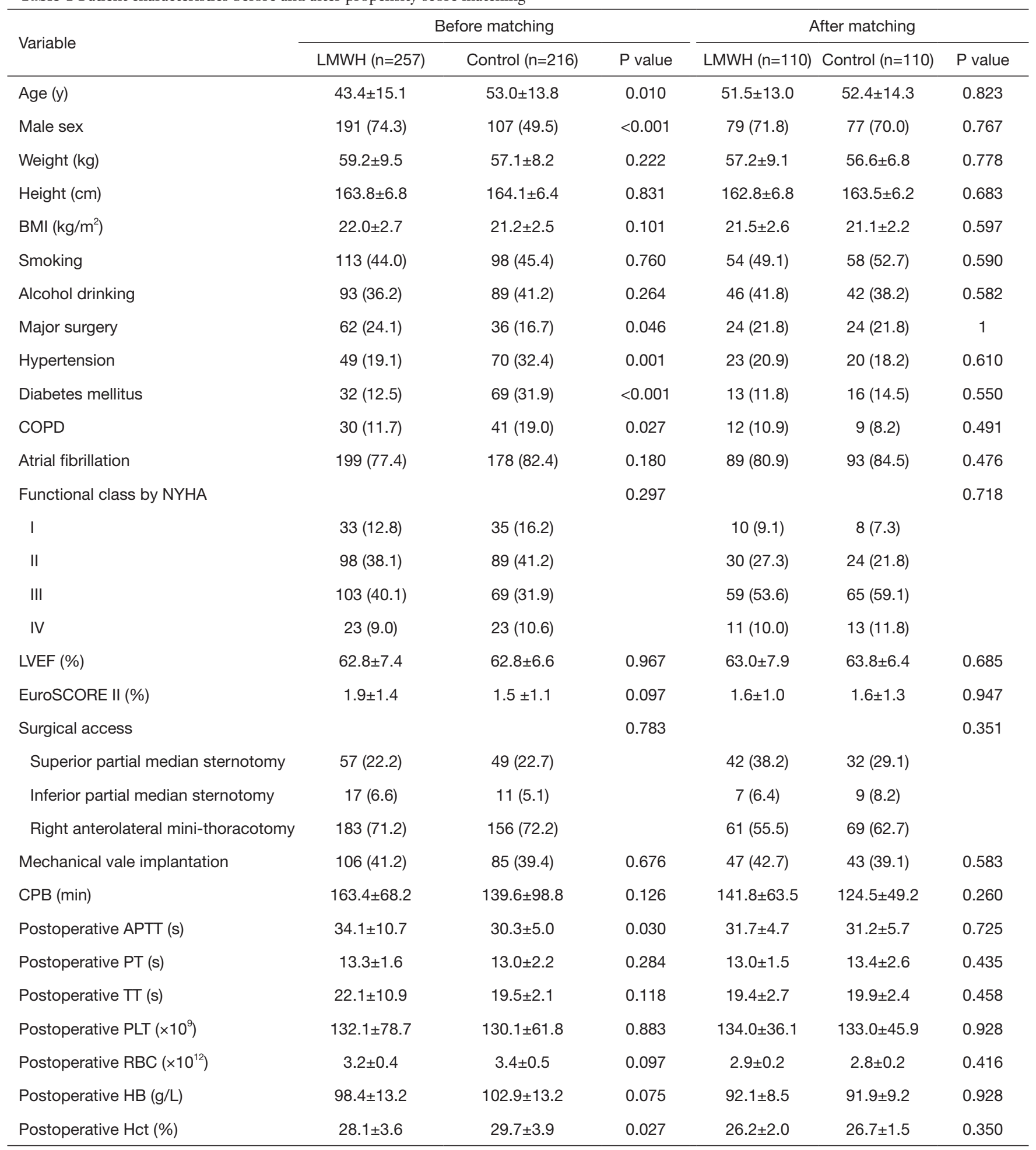

In the matched cohorts, no significant covariate difference was found between the two groups. BMI, body mass index; COPD, chronic obstructive pulmonary disease; NYHA, New York Heart Association; LVEF, left ventricular ejection fractions; EuroSCORE II, European system for cardiac operative risk evaluation II; CPB, cardiopulmonary bypass; APTT, activated partial thromboplastin time; PT, prothrombin time; TT, thrombin time; PLT, platelet counts; RBC, red blood cell counts; HB, hemoglobin; Hct, hematocrit. 
group and 17 major bleeding events (5 hemopericardium and 12 hemothorax) in the Control group (Table 2).

After matching, no embolism event was observed in either group, while there was a significant higher rate of major bleeding events in the LMWH group than in the Control group $(14.5 \%$ vs. $5.5 \%, \mathrm{P}=0.025)$. There were 16 major

Table 2 Embolism events and major bleeding events before propensity score matching

\begin{tabular}{lccc}
\hline Variable & LMWH $(\mathrm{n}=257)$ Control $(\mathrm{n}=216)$ & $\mathrm{P}$ value \\
\hline Embolic events (\%) & $1(0.4)$ & $3(1.4)$ & 1.000 \\
DVT & 1 & 1 & \\
Shock & 0 & 2 & \\
Major bleeding events (\%) & $41(16.0)$ & $17(7.9)$ & 0.008 \\
Hemopericardium & 14 & 5 & \\
Hemothorax & 27 & 12 & \\
\hline
\end{tabular}

Before PSM, there were fewer embolic events in both groups though the difference was not statistically significant, and there was a significant higher rate of major bleeding events in the LMWH group than in the Control group. DVT, deep vein thrombosis. bleeding events ( 7 hemopericardium and 9 hemothorax) in the LMWH group and 6 major bleeding events ( 2 hemopericardium and 4 hemothorax) in the Control group. There was no significant difference in the total volume of red blood cell transfusion between the two groups $(400.9 \pm 295.7$ vs. $378.2 \pm 270.0 \mathrm{~mL}, \mathrm{P}=0.552)$. Postoperative drainage tube indwelling time in LMWH group was significantly longer than that in Control group $(3.9 \pm 1.0$ vs. $2.5 \pm 0.6$ days, $\mathrm{P}<0.001)$. Postoperative total volume of drainage was significantly increasing in the LMWH group $(680.2 \pm 89.8$ vs. $302.5 \pm 79.9 \mathrm{~mL}, \mathrm{P}<0.001)$. There was no significant difference in assisted mechanical ventilation time $(8.7 \pm 4.8$ vs. $8.4 \pm 3.5$ hours, $\mathrm{P}=0.542)$ and ICU stay time ( $2.4 \pm 1.0$ vs. $2.2 \pm 0.9$ days, $\mathrm{P}=0.166)$ between the two groups. However, the hospital stay time of the LMWH group was significantly longer $(9.5 \pm 1.8$ vs. $7.0 \pm 1.6$ days, $\mathrm{P}<0.001)$. The LMWH group also had significantly higher incidence of impaired wound healing $(16.4 \%$ vs. $5.5 \%, \mathrm{P}=0.009)$ (Table 3).

\section{Discussion}

MICS surgery represents technical innovations within the field of cardiac surgery (23). Peripheral cardiopulmonary

Table 3 Short term outcome after propensity score matching

\begin{tabular}{|c|c|c|c|}
\hline Variable & LMWH $(n=110)$ & Control $(n=110)$ & $P$ value \\
\hline Duration of chest tube placement (days) & $3.9 \pm 1.0$ & $2.5 \pm 0.6$ & $<0.001$ \\
\hline The total volume of postoperative drainage $(\mathrm{mL})$ & $680.2 \pm 89.8$ & $302.5 \pm 79.9$ & $<0.001$ \\
\hline Ventilation time (hours) & $8.7 \pm 4.8$ & $8.4 \pm 3.5$ & 0.542 \\
\hline Postoperative hospital stay (days) & $9.5 \pm 1.8$ & $7.0 \pm 1.6$ & $<0.001$ \\
\hline Embolic events & 0 & 0 & $\mathrm{~N} / \mathrm{A}$ \\
\hline DVT & 0 & 0 & \\
\hline Shock & 0 & 0 & \\
\hline Hemothorax & 9 & 4 & \\
\hline Poor wound healing (\%) & $18(16.4)$ & $6(5.5)$ & 0.009 \\
\hline
\end{tabular}

After matching, no embolism event was observed in either group, while there was a significant higher rate of major bleeding events in the LMWH group than in the Control group. Postoperative drainage tube indwelling time in LMWH group was significantly longer than that in Control group. Postoperative total volume of drainage was significantly increasing in the LMWH group. The postoperative hospital stay time of the LMWH group was significantly longer. The LMWH group had significantly higher incidence of impaired wound healing. DVT, deep vein thrombosis. 
bypass with femoral cannulation is currently the most commonly used method in MICS (24). Although it offers many advantages over conventional surgery (3-5), there are few studies reporting the complications associate with these procedures (25), there were no study research whether it would increase the risk of VTE and there has been no consensus about whether to apply with LMWH for early prophylactic anticoagulation among MICS patients (9). To our knowledge, this is the first report using retrospective PSM cohorts to assess the benefits and possible harms of early prophylactic LMWH postoperatively in patients underwent MICS.

Aziz and colleague report incidence of DVT after cardiac surgery was $2.07 \%$ (10). Our research results are no higher than theirs, which may mean that MICS is not a risk factor for venous thromboembolism. There is evidence that a prothrombotic state is common after cardiac surgery (26). According to the European Association for Cardiothoracic Surgery guidelines, prophylactic anti-coagulation for VTE should be commenced from the first postoperative day (13). LMWH seems to offer effective and stable anticoagulation and has been used in observational series (27-30). This study demonstrated that early prophylactic anticoagulation with LMWH did not significantly reduce the incidence of embolism events, while increased the incidence of bleeding events. The early postoperative use of LMWH increased the total volume of drainage, thereby delaying the removal of chest tube, and early postoperative use of LMWH did not benefit MICS patients at clinical endpoints, even if most patients were at high risk of thrombosis [Caprini Score (31) greater than 5 points]. The injury of venous endothelial during MICS did not increase the incidence of DVT, we assumed it had been compensated by early ambulant after MICS although many patients had a high Caprini Score. In addition, some researches showed that the Chinese population had lower risk of thrombotic events but higher risk of bleeding events compared with western populations (32-34). Our findings revealed that early use of LMWH for anticoagulation might increase the total volume of drainage and chest tube indwelling time.

Moreover, early use of LMWH for anticoagulation might increase the incidence of pericardial effusion and pleural effusion, comparing to control group, the incidence of delayed postoperative hemopericardium and hemothorax is significantly higher in LMWH group, which might lead to longer hospitalization. Besides, higher incidence of impaired wound healing in LMWH group might be contributed by to heparin-related subcutaneous bleeding.

\section{Limitations}

Our retrospective study presents several limitations. First, this was a single-center study with a relatively small sample size, which resulted in low incidence of embolic events. Second, all enrolled patients in this study underwent valve surgery, and valvular heart disease usually had a long history and congestive heart failure, that often led to underlying preoperative liver dysfunction, which cause these patients had higher risk of bleeding than embolism events, and that could be one reason for little benefit of early postoperative administration of LMWH in MICS patients. Due to few patients suffered from coronary heart disease undergoing minimally invasive bypass surgery in our hospital, whether postoperative injection of $\mathrm{LMWH}$ could benefit these patients remained unknown.

\section{Conclusions}

Early prophylactic anticoagulation with LMWH could not reduce the incidence of embolism events after MICS. Instead, it might increase postoperative bleeding events and extend drainage tube indwelling time and the length of hospital stay. We need more evidence to support the use of LMWH for VTE prophylaxis in patients underwent MICS.

\section{Acknowledgments}

Funding: This work was supported by the National Nature Science Foundation of China (No. 81300102, No. 81170232, No. 81270419 and No. 81770244), Science Foundation of Shanghai Municipal Commission of Health and Family Planning (No. 201740225) and Shanghai Shenkang Emerging Advanced Technology Project (SHDC12014107).

\section{Footnote}

Conflicts of Interest: The authors have no conflicts of interest to declare.

Ethical Statement: The authors are accountable for all aspects of the work in ensuring that questions related to the accuracy or integrity of any part of the work are appropriately investigated and resolved. The study protocol was approved by Committee on Ethic of Biomedicine Research, Second Military Medical University (No. 2011SL037), and met the ethical and legal requirements. 
All subjects have signed informed consent for surgery, perioperative therapy and related medical research.

\section{References}

1. Nkomo VT, Gardin JM, Skelton TN, et al. Burden of valvular heart diseases: a population-based study. Lancet 2006;368:1005-11.

2. Baumgartner H, Falk V, Bax JJ, et al. 2017 ESC/EACTS Guidelines for the Management of Valvular Heart Disease. Rev Esp Cardiol (Engl Ed) 2018;71:110.

3. Li W, Xue Q, Liu K, et al. Effects of MIAVS on Early Postoperative ELWI and Respiratory Mechanics. Med Sci Monit 2016;22:1085-92.

4. Dieberg G, Smart NA, King N. Minimally invasive cardiac surgery: A systematic review and meta-analysis. Int J Cardiol 2016;223:554-60.

5. Schmitto JD, Mokashi SA, Cohn LH. Minimally-invasive valve surgery. J Am Coll Cardiol 2010;56:455-62.

6. Chan EY, Lumbao DM, Iribarne A, et al. Evolution of cannulation techniques for minimally invasive cardiac surgery: a 10-year journey. Innovations (Phila) 2012;7:9-14.

7. Ramchandani M, Al Jabbari O, Abu Saleh WK, et al. Cannulation Strategies and Pitfalls in Minimally Invasive Cardiac Surgery. Methodist Debakey Cardiovasc J 2016;12:10-3.

8. Reitsma PH, Versteeg HH, Middeldorp S. Mechanistic view of risk factors for venous thromboembolism. Arterioscler Thromb Vasc Biol 2012;32:563-8.

9. Ho KM, Bham E, Pavey W. Incidence of Venous Thromboembolism and Benefits and Risks of Thromboprophylaxis After Cardiac Surgery: A Systematic Review and Meta-Analysis. J Am Heart Assoc 2015;4:e002652.

10. Aziz F, Patel M, Ortenzi G, et al. Incidence of Postoperative Deep Venous Thrombosis Is Higher among Cardiac and Vascular Surgery Patients as Compared with General Surgery Patients. Ann Vasc Surg 2015;29:661-9.

11. Viana VB, Melo ER, Terra-Filho M, et al. Frequency of Deep Vein Thrombosis and/or Pulmonary Embolism After Coronary Artery Bypass Grafting Investigation Regardless of Clinical Suspicion. Am J Cardiol 2017;119:237-42.

12. Di Nisio M, Peinemann F, Porreca E, et al. Primary prophylaxis for venous thromboembolism in patients undergoing cardiac or thoracic surgery. Cochrane Database Syst Rev 2015;(6):CD00965.

13. Dunning J, Versteegh M, Fabbri A, et al. Guideline on antiplatelet and anticoagulation management in cardiac surgery. Eur J Cardiothorac Surg 2008;34:73-92.

14. Gould MK, Garcia DA, Wren SM, et al. Prevention of VTE in nonorthopedic surgical patients: Antithrombotic Therapy and Prevention of Thrombosis, 9th ed: American College of Chest Physicians Evidence-Based Clinical Practice Guidelines. Chest 2012;141:e227S-77S.

15. Cikirikcioglu M, Myers PO, Kalangos A. First do no harm: postoperative thromboprophylaxis following open heart surgery. Eur J Cardiothorac Surg 2013;44:184.

16. Kearon C, Akl EA, Ornelas J, et al. Antithrombotic Therapy for VTE Disease: CHEST Guideline and Expert Panel Report. Chest 2016;149:315-52.

17. Streiff MB, Agnelli G, Connors JM, et al. Erratum to: Guidance for the treatment of deep vein thrombosis and pulmonary embolism. J Thromb Thrombolysis 2016;41:548.

18. Hirsh J, Warkentin TE, Shaughnessy SG, et al. Heparin and low-molecular-weight heparin: mechanisms of action, pharmacokinetics, dosing, monitoring, efficacy, and safety. Chest 2001;119:64S-94S.

19. Weitz JI. Low-molecular-weight heparins. N Engl J Med 1997;337:688-98.

20. Nashef SA, Roques F, Sharples LD, et al. EuroSCORE II. Eur J Cardiothorac Surg 2012;41:734-44; discussion 744-5.

21. Zhang Y, Liu K, Li W, et al. An Adjusted Calculation Model of Reduced Heparin Doses in Cardiopulmonary Bypass Surgery in a Chinese Population. J Cardiothorac Vasc Anesth 2016;30:1179-83.

22. D'Agostino RB, Jr. Propensity score methods for bias reduction in the comparison of a treatment to a nonrandomized control group. Stat Med 1998;17:2265-81.

23. del Nido PJ. Surgical Innovation: Lessons From the Pragmatic Philosophical School. Ann Thorac Surg 2015;100:778-83.

24. Shann K, Melnitchouk S. Advances in Perfusion Techniques: Minimally Invasive Procedures. Semin Cardiothorac Vasc Anesth 2014;18:146-52.

25. Lamelas J, Williams RF, Mawad M, et al. Complications Associated With Femoral Cannulation During Minimally Invasive Cardiac Surgery. Ann Thorac Surg 2017;103:1927-32.

26. Parolari A, Mussoni L, Frigerio M, et al. Increased prothrombotic state lasting as long as one month after on-pump and off-pump coronary surgery. J Thorac Cardiovasc Surg 2005;130:303-8.

27. Rivas-Gándara N, Ferreira-Gonzalez I, Tornos P, et al. Enoxaparin as bridging anticoagulant treatment in cardiac 
surgery. Heart 2008;94:205-10.

28. Meurin P, Tabet JY, Weber H, et al. Low-molecular-weight heparin as a bridging anticoagulant early after mechanical heart valve replacement. Circulation 2006;113:564-9.

29. Bucci C, Geerts WH, Sinclair A, et al. Comparison of the effectiveness and safety of low-molecular weight heparin versus unfractionated heparin anticoagulation after heart valve surgery. Am J Cardiol 2011;107:591-4.

30. Fanikos J, Tsilimingras K, Kucher N, et al. Comparison of efficacy, safety, and cost of low-molecular-weight heparin with continuous-infusion unfractionated heparin for initiation of anticoagulation after mechanical prosthetic valve implantation. Am J Cardiol 2004;93:247-50.

Cite this article as: Li W, Wang P, Le S, Xi W, Wang J, Yin L, Wang Q, Zhang Y, Wang Z. Benefits may not outweigh risks of low molecular weight heparin $(\mathrm{LMWH})$ in early postoperative thromboprophylaxis following minimally invasive cardiac surgery: a propensity score-matched analysis. J Thorac Dis 2019;11(12):5266-5273. doi: 10.21037/jtd.2019.11.56
31. Caprini JA. Risk assessment as a guide to thrombosis prophylaxis. Curr Opin Pulm Med 2010;16:448-52.

32. Jorgensen AL, FitzGerald RJ, Oyee J, et al. Influence of CYP2C9 and VKORC1 on patient response to warfarin: a systematic review and meta-analysis. PLoS One 2012;7:e44064.

33. Yang J, Chen Y, Li X, et al. Influence of CYP2C9 and VKORC1 genotypes on the risk of hemorrhagic complications in warfarin-treated patients: a systematic review and meta-analysis. Int J Cardiol 2013;168:4234-43.

34. Tang L, Wang HF, Lu X, et al. Common genetic risk factors for venous thrombosis in the Chinese population. Am J Hum Genet 2013;92:177-87. 